\title{
Sirtuin-3 (SIRT3), a therapeutic target with oncogenic and tumor-suppressive function in cancer
}

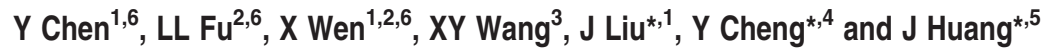

Sirtuin-3 (SIRT3), a major mitochondria NAD +-dependent deacetylase, may target mitochondrial proteins for lysine deacetylation and also regulate cellular functions. And, SIRT3 is an emerging instrumental regulator of the mitochondrial adaptive response to stress, such as metabolic reprogramming and antioxidant defense mechanisms. Accumulating evidence has recently demonstrated that SIRT3 may function as either oncogene or tumor suppressor on influencing cell death by targeting a series of key modulators and their relevant pathways in cancer. Thus, in this review, we present the structure, transcriptional regulation, and posttranslational modifications of SIRT3. Subsequently, we focus on highlighting the Janus role of SIRT3 with oncogenic or tumor-suppressive function in cancer, which may provide more new clues for exploring SIRT3 as a therapeutic target for drug discovery.

Cell Death and Disease (2014) 5, e1047; doi:10.1038/cddis.2014.14; published online 6 February 2014

Subject Category: Cancer

Facts

- SIRT3, a major mitochondrial $\mathrm{NAD}^{+}$-dependent deacetylase, has a key role in cancer.

- The function of SIRT3 may be different depending on the cell-type and tumor-type; thereby, SIRT3 may act as an oncogene or a tumor suppressor.

- Targeting SIRT3 would be a promising therapeutic strategy for the discovery of more activators or inhibitors in cancer.

\section{Open Questions}

- How sirtuin-3 (SIRT3) can be involved in the hallmarks of cancer?

- Why does SIRT3 have either pro-survival (oncogenic) or pro-death (tumor-suppressive) role in cancer?

- Whether would targeting SIRT3 be a novel cancer therapeutic strategy?

Initially classified as class III histone deacetylases, sirtuins (SIRTs), referring to seven SIRTs (SIRT1-7), are now known to possess diverse enzymatic activities and regulate a wide range of intracellular processes including metabolism, longevity, aging, cancer and response to stress. ${ }^{1,2}$ These proteins are characterized by an evolutionarily conserved SIRT core domain that contains the catalytic and $\mathrm{NAD}^{+}$binding domain. ${ }^{1}$ Among them, SIRT3 is an important mitochondrial protein, which may function as a primary mitochondrial stressresponsive protein deacetylase..$^{2,3}$

SIRT3 has been found to regulate many aspects of mitochondrial function, such as metabolism, ATP generation and modulation of the response to oxidative stress. The first substrate identified for SIRT3, acetyl-CoA synthetase 2 (AceCS2) and glutamate dehydrogenase (GDH) have shown that SIRT3 may have a regulatory role in mitochondrial metabolism as AceCS2 localizes to the mitochondria, and is activated by deacetylation. ${ }^{4}$ SIRT3-dependent deacetylation also increases the enzymatic activity of GDH, which is involved in the oxidation of amino acids. ${ }^{5}$ Interestingly, a shift from dependence on liver glycolysis, facilitated by $\mathrm{GDH}$ and AceCS2 activity, has been implicated in calorie restriction (CR), suggesting a role of SIRT3 in reprogramming metabolism during CR to allow respiration. SIRT3 further modulates energy homeostasis in the mitochondria by regulating ATP generation from oxidative phosphorylation. Mechanistically,

\footnotetext{
${ }^{1}$ State Key Laboratory of Biotherapy, Department of Gastrointestinal Surgery, West China Hospital, Sichuan University, Chengdu 610041, China; ${ }^{2}$ College of Life Sciences, Sichuan University, Chengdu 610064, China; ${ }^{3}$ Analytical \& Testing Center, Sichuan University, Chengdu 610064, China; ${ }^{4}$ Department of Pharmacology and The Penn State Cancer Institute, The Pennsylvania State University College of Medicine, Milton S Hershey Medical Center, Hershey, Pennsylvania, USA and ${ }^{5}$ School of Traditional Chinese Materia Medica, Shenyang Pharmaceutical University, Shenyang 110016, China

${ }^{*}$ Corresponding author: J Liu, Y Cheng and J Huang, State Key Laboratory of Biotherapy, Sichuan University, Ke-yuan Road 4, No 1, Gao-peng Street, Chengdu, Sichuan 610041, China. Tel/Fax: +86 28 85503817; E-mail: liujie2011 @scu.edu.cn (JL) or yxc24@psu.edu (YC) or 13504051049@ 163.com (JH)

${ }^{6}$ These authors contributed equally to this work.

Keywords: sirtuin-3 (SIRT3); mitochondria; oncogene; tumor suppressor; therapeutic target

Abbreviations: AceCS2, acetyl-CoA synthetase 2; CR, calorie restriction; ERR, estrogen-related receptor; ETC, electron transport chain; FAK, focal adhesion kinase; GC, gastric cancer; GDH, glutamate dehydrogenase; HCC, hepatocellular carcinoma; HK II, hexokinase II; HIF-1 $\alpha$, hypoxia-inducible factor 1 $\alpha$; JNK2, c-Jun N-terminal kinase 2; MPT, mitochondrial permeability transition; NMNAT2, nicotinamide mononucleotide adenylyltransferase 2; OSCC, oral squamous cell carcinoma; PPARc, peroxisome proliferator-activated receptor c; RIP, Receptor-interacting protein; ROS, reactive oxygen species; SIRT3, sirtuin-3; SOD2, superoxide dismutase 2; Tam, tamoxifen; TCA, tricarboxylic acid cycle

Received 20.11.13; revised 05.1.14; accepted 07.1.14; Edited by A Stephanou
} 
SIRT3 may interact with subunits of complexes I and II of the electron transport chain (ETC), leading to increased activity of both complexes. ${ }^{6}$ The mitochondrial ribosomal protein MRPL10, required for the synthesis of proteins encoded by mitochondrial DNA, has been identified as a SIRT3 target, revealing a regulatory role for SIRT3 in mitochondrial respiration. ${ }^{7}$ In addition, SIRT3 mediates cellular resistance toward various forms of stress by maintaining genomic stability and mitochondrial integrity. For example, SITR3 may influence cell survival by regulating the state of the mitochondrial permeability transition (MPT), owing to the fact that inhibition of SIRT3 activity enhances the sensitivity of mitochondria to induction of MPT by increasing cyclophilin D activity. ${ }^{8}$ As mentioned above, it has revealed a complex role for SIRT3 in the regulation of mitochondrial activity, with involvement in respiration, ETC and MPT.

SIRT3 is central to the maintenance of appropriate mitochondrial function by limiting oxidative stress, and reducing reactive oxygen species (ROS) production with a decrease in mitochondrial membrane potential. ${ }^{9}$ The expression of two antioxidant proteins including mitochondrial superoxide dismutase 2 (SOD2) and catalase is increased by overexpression of SIRT3. ${ }^{10}$ FoxO3a, a transcriptional factor that upregulates SOD2 and catalase, can be deacetylated by SIRT3, providing a mechanism by which SIRT3 decreases ROS levels. ${ }^{10}$ Given the facts that SIRT3 localizes to mitochondria and that mitochondrial ROS are important in cancer, SIRT3 may have a key role in carcinogenesis. By deacetylating proteins involved in multiple mitochondrial processes, SIRT3 can coordinate global shifts in mitochondrial activity, thereby indicating some implications for tumor proliferation. ${ }^{11}$ Loss of SIRT3 triggers oxidative damage, ROS-mediated signaling and metabolic reprogramming that can work together to lead to carcinogenesis. On one hand, SIRT3 is pro-survival and prevents cell death in response to stress and starvation. ${ }^{11}$ In addition, it can protect from cell death in response to hypoxia or the apoptotic inducer staurosporine. On the other hand, SIRT3 is pro-apoptotic under certain pathway conditions. ${ }^{12}$ SIRT3 has been identified as a tumor suppressor, which directly links SIRT3 to metabolic processes. SIRT3 seems to be involved in carcinogenesis; thereby, SIRT3 inhibitors/activators might have some potential therapeutic benefits. ${ }^{13}$

\section{SIRT3 Structure}

SIRT3 contains a conserved enzymatic core with two domains, including a large Rossmann fold domain that binds $\mathrm{NAD}^{+}$and a small domain formed by two insertions of the large domain binding to a zinc atom. The acetylated peptide substrate binds to the cleft between the two domains. ${ }^{14} \mathrm{~A}$ few key enzymes involved in energy production in the mitochondria have been identified as SIRT3 substrates. Deacetylation of AceCS2 at lysine 642 by SIRT3 activates AceCS2 activity, providing increased acetyl-CoA to feed into the tricarboxylic acid cycle (TCA). ${ }^{15}$ The cofactor-binding pocket can be divided into three regions: the adenine ribose moiety of $\mathrm{NAD}^{+}$, the nicotinamide ribose moiety and the catalytic center located deep inside the pocket. Structural evidence suggests that the binding of acetyl-lysine positions the nicotinamide group of $\mathrm{NAD}^{+}$within a highly conserved pocket where the carboxamide of $\mathrm{NAD}^{+}$interacts with a conserved aspartate residue. ${ }^{16}$ The crystal structure of SIRT3 with acetylated AceCS2 peptide has been obtained to show that SIRT3 can form a stable complex with the substrate peptide in the absence of $\mathrm{NAD}^{+}$. Also, resveratrol and oroxylin $\mathrm{A}$ (activators) and 5-amino-2-phenyl-benzoxazole (inhibitor) have been reported to bind to SIRT3 (Figure 1).

\section{Expression, Transcriptional Regulation and Post- translational Modifications of SIRT3}

SIRT3 resides primarily in the mitochondria, binding and deacetylating several metabolic enzymes that regulate important mitochondrial functions. It has been shown that SIRT3 can particularly regulate production of ROS at the ETC. ${ }^{17,18}$ Further, SIRT3 is linked to the metabolic reprogramming termed the Warburg effect in cancer cells, which enforces the glycolytic production of ATP. ${ }^{19}$ Recent studies have highlighted the ability of SIRT3 to protect cells from oxidative damage, implicating an important role of SIRT3 in regulating ROS homeostasis. ${ }^{20}$ Moreover, mitochondria of tumor cells can exhibit aberrant ROS, accounting for the high degree of genomic instability demonstrated in cancer cells. SIRT3 can protect cells against ROS by enhancing the activity of antioxidant defense system, suggesting that this protein can be a crucial mitochondrial fidelity protein in response to oxidative stress and loss-of-function of SIRT3. ${ }^{21}$ The ROS that are produced when SIRT3 activity is suppressed activate nuclear hypoxia-inducible factor $1 \alpha(\mathrm{HIF}-1 \alpha)$, which turns on genes for glycolysis and angiogenesis. Indeed, the enforced SIRT3 expression may reverse the Warburg effect in many tumor cells. ${ }^{22}$ In addition, it is now well established that SIRT3 deacetylation activity is activated by $\mathrm{CR}$, and this induction in deacetylation activity also appears to protect against the development of cancer induction. ${ }^{23}$ Calorie-restricted rodents show attenuation of age-associated increases in mitochondrial generation of $\mathrm{O}_{2}^{-}$and $\mathrm{H}_{2} \mathrm{O}_{2}$, and slower accumulation of oxidative damage. ${ }^{24}$ Notably, SIRT3 expression is increased during $\mathrm{CR}$ and consequently mitochondrial lysine acetylation is altered in a tissue-specific manner, indicating a role for SIRT3 in regulating mitochondrial function in response to CR through deacetylation of mitochondrial target proteins. ${ }^{25}$

SIRT3 is a mitochondrial protein, with its N-terminal 25 amino-acid residues that are responsible for its mitochondrial localizaion. ${ }^{26}$ Also, SIRT3 is synthesized as an inactive protein and also activated by matrix peptidase. ${ }^{27}$ Peroxisome proliferator-activated receptor c (PPARc) coactivator-1 $\alpha$ (PGC-1 $\alpha$ ) can powerfully stimulate SIRT3 gene expression, mediated by an estrogen-related receptor (ERR)-binding element mapped to the SIRT3 promoter region. PGC-1 $\alpha$ stimulates SIRT3 expression at both mRNA and protein levels, and the cotransfection of PGC- $1 \alpha$ and $E R R \alpha$ has a synergic effect on SIRT3 promoter activity. ${ }^{28}$ Moreover, PGC-1 $\alpha$ induces SIRT3 expression through coactivation of ERR $\alpha$, and in turn, SIRT3 stimulates PGC-1 $\alpha$ gene expression through activation of CREB phosphorylation, thus forming a positive-feedback loop. In addition to suppression of basal ROS production in cells, SIRT3 mediates the induction of ROS-detoxifying enzymes and reduction of ROS level by 

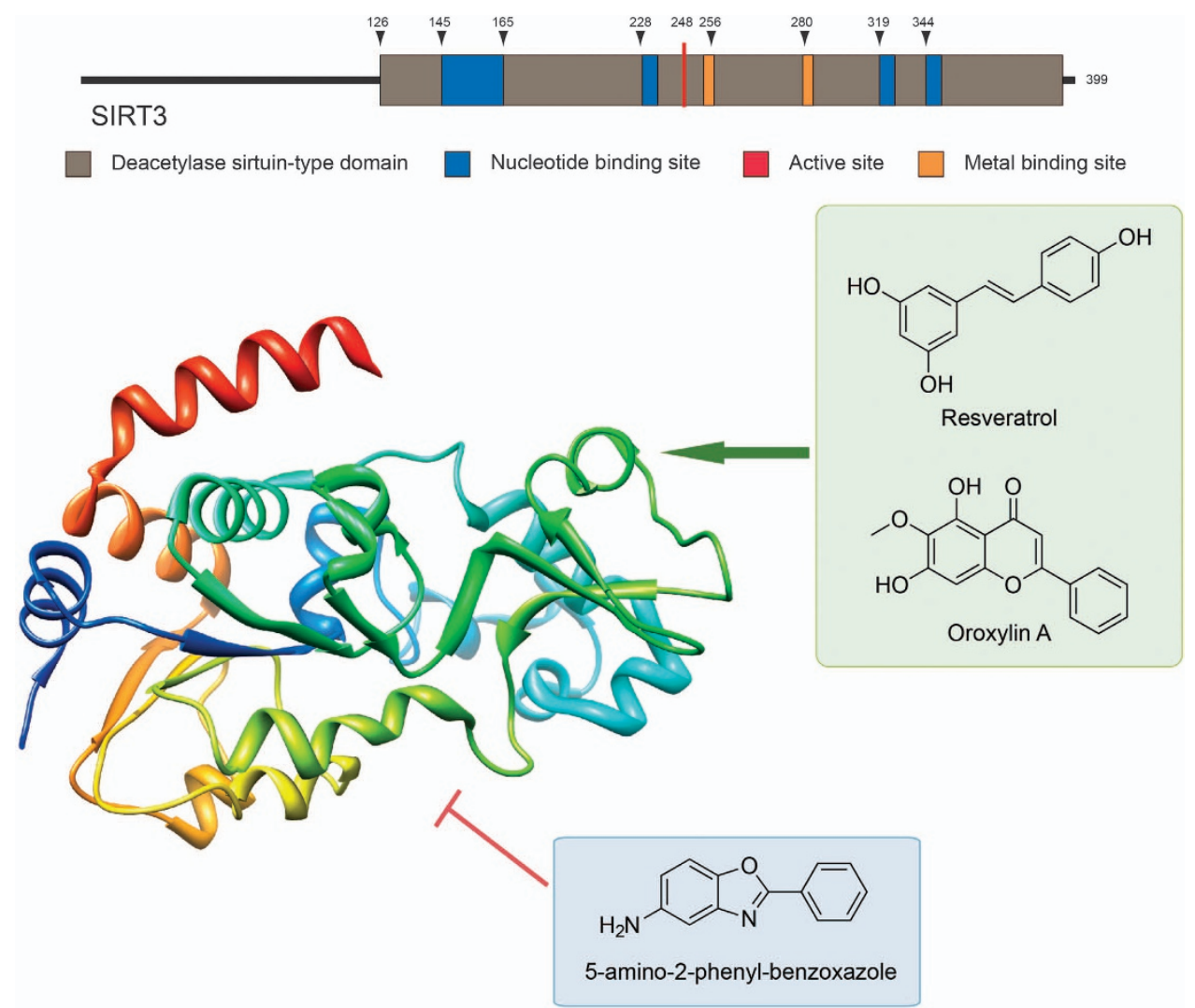

Figure 1 SIRT3 structure with its activators and inhibitors. SIRT3 is a soluble protein located in the mitochondrial matrix, containing a mitochondrial processing peptide at the N-terminus, with a conserved enzymatic core with two domains. Moreover, several SIRT3 activators (resveratrol and oroxylin A) and novel SIRT3 inhibitor 5-amino-2-phenyl-benzoxazole have also been reported

PGC-1 $\alpha .{ }^{29}$ Acetyl-CoA is well known to be a primary entry point for nutrient catabolism by the TCA cycle, and thus NAD is a major electron acceptor for multiple metabolic reactions. ${ }^{30}$ Moreover, lysine acetylation is regulated by SIRT3 because acetyltransferases can use acetyl-CoA as an acetyl donor and SIRT3 can use NAD for deacetylation.

\section{SIRT3 and the Hallmarks of Cancer}

SIRT3 has been a major focus of study in the scientific community on understanding cancer, one of the leading causes of death worldwide, as the role of SIRT3 is identified in the hallmarks of cancer. ${ }^{31}$ Cancerous cells possess six hallmarks that include self-sufficiency in growth signals, insensitivity to antigrowth signals, resisting cell death, sustained angiogenesis, limitless replicative potential, and tissue invasion and metastasis. ${ }^{32}$ Additional four characteristics that have recently emerged include dysregulation of cellular energy, avoidance of immune distraction, genomic instability and tumor-promoting inflammation. Interestingly, growing evidence has supported a crucial role for SIRT3 with some hallmarks of cancer (Figure 2). Direct links between SIRT3 and genomic stability have been demonstrated that SIRT3-mediated ROS may contribute to genomic instability. Moreover, SIRT3 seems to regulate ROS through MnSOD and SOD2 modulation, associated with mutagenesis promotion and genomic instability. ${ }^{33}$ Additional studies that support nuclear localization for SIRT3 upon cellular stress have suggested that histone modifications H4K16Ac and H3K9Ac are targeted by SIRT3, indicating the possible role of SIRT3 in genomic stability. ${ }^{34}$ The role of SIRT3 in cellular energetics and tumorigenesis has also been extensively discussed, as SIRT3 mediates metabolic reprogramming by destabilizing $\mathrm{HIF}-1 \alpha$, and then regulates glycoytic gene expression. Under hypoxic conditions, SIRT3 overexpression inhibits ROS production, glycolysis, proliferation as well as HIF- $1 \alpha$ stabilization and its downstream transcriptional activity, thereby decreasing tumorigenesis. ${ }^{35}$ Moreover, the link among mitochondrial functions, inflammation and cancer has been well documented in lung and colorectal cancers. SIRT3 positively regulates antioxidant gene expression, thus decreasing ROS accumulation, thereby leading to decreased inflammation. ${ }^{36}$ In addition, activation of the reninangiotensin system, ACE/Ang II/AT1R, is associated with downregulation of the pro-survival genes Nampt and SIRT3, increase in ROS production, and pro-inflammatory cytokine and chemokine releases, leading to inflammation and autoimmune dysfunction. ${ }^{36,37}$

Moreover, carcinogenesis is associated with the metabolic reprogramming of cells, orchestrated by various mechanisms including transcriptional changes induced by the transcription factor HIF-1 $\alpha$. Upon stabilization, HIF-1 $1 \alpha$ can translocate to the nucleus and induce the transcription of its target genes especially in cancer. ${ }^{38}$ SIRT3 has been demonstrated to limit 


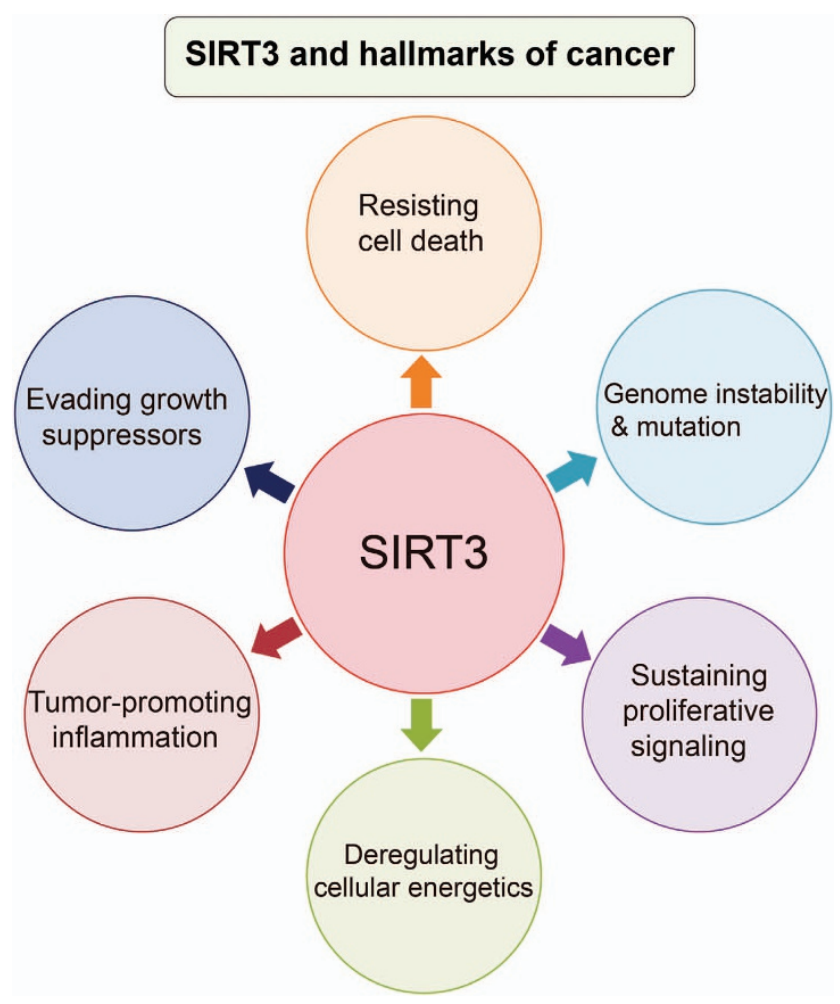

Figure 2 SIRT3 and the hallmarks of cancer. SIRT3 can link to six hallmarks of cancer, such as resisting cell death, genomic instability and mutation, sustaining proliferative signaling, dysregulation of cellular energetics, tumor-promoting inflammation and invading growth suppressors

ROS levels, thereby leading to the destabilization and subsequent degradation of HIF- $1 \alpha{ }^{39}$ Moreover, by preventing intracellular acidification, SIRT3 can inhibit mitochondrial translocation of pro-apoptotic protein Bax, and maintain mitochondrial membrane integrity. ${ }^{40}$ Intracellular acidification followed by mitochondrial accumulation of Bax is more evident in hypoxic SIRT3-silenced cells, and by contrast, SIRT3 overexpression may prevent intracellular acidification, Bax activation and MPT induction. Interestingly, intracellular acidification is important in tumor progression and resistance to treatment, which is related to HIF-1 $\alpha$ activation (Figure 3 ).

\section{SIRT3 Functions as an Oncogene}

SIRT3 is critical for maintaining mitochondrial integrity and function, which can regulate cell survival, death and metabolic pathways, helping to control the balance between health and disease. ${ }^{41}$ With its central role in mitochondrial biology, SIRT3 contributes to cell survival by modulating oxidative stress pathways. In head and neck squamous cell carcinoma where SIRT3 is overexpressed, SIRT3 can maintain ROS levels at the appropriate levels for maintaining a proliferative and aggressive phenotype, thus preventing apoptosis and promoting carcinogenesis. ${ }^{42}$ SIRT3 is also a stress-responsive deacetylase, whose increased expression may protect cancer cells from genotoxic and oxidative stress-mediated cell death. By binding to and deacetylating Ku70, SIRT3 augments Ku70-Bax interactions and prevents Bax translocation to the mitochondria as well as apoptosis during stress-mediated

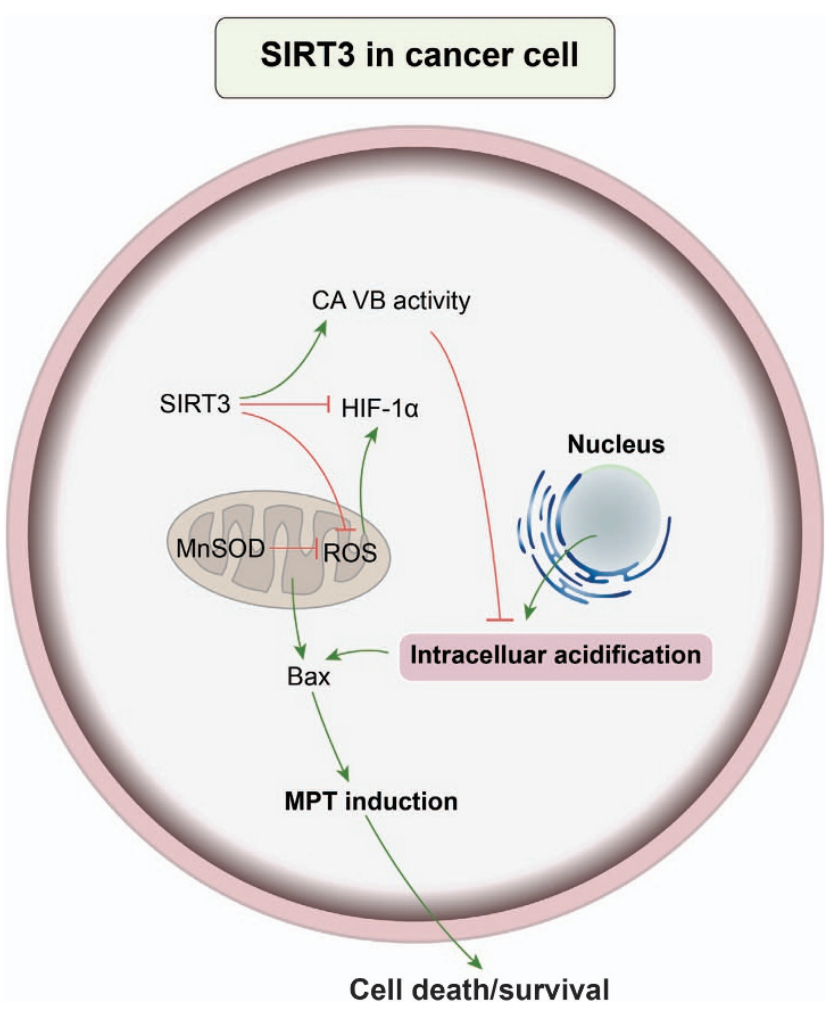

Figure 3 SIRT3 in cancer cell. SIRT3 can limit ROS levels in cancer cell, thereby leading to the destabilization and subsequent degradation of HIF-1 $\alpha$. Moreover, SIRT3 overexpression may prevent intracellular acidification, Bax activation and MPT induction

conditions in HeLa cells. ${ }^{43}$ Further, SIRT3 appears coupled with the tumor suppressor p53 during the initiation of its expression in the mitochondria, usually by abrogating p53 activity to enact growth arrest and senescence. Subsequently, p53 is regarded as a new target for SIRT3 deacetylation in bladder cancer as SIRT3 can rescue p53induced growth arrest in human bladder tumor-derived EJ-p53 cells. ${ }^{44}$ Cumulative mitochondrial damage leads to a fall in relative NAD levels and a concomitant fall in SIRT3 activity, which is also associated with growth arrest, senescence and apoptosis. In addition, SIRT3 alters sensitivity of breast cancer cells to tamoxifen (Tam), a commonly used antiestrogen agent. In sensitive MCF-7 cells, SIRT3 can be significantly increased following exposure to Tam, and transfection of MCF-7 cells with a SIRT3 expression plasmid may decrease cellular sensitivity to Tam and block the Taminduced apoptosis. Thus, SIRT3 is considered as a potential target for overcoming Tam resistance in the treatment of breast cancer. ${ }^{45}$ Also, SIRT3 has been immune-stained in some samples and semiquantified using the HSCORE method to evaluate the predictive value of SIRT3 expression levels on esophageal cancer outcome, and the level of SIRT3 expression is demonstrated as an independent predictor for esophageal cancer outcome. ${ }^{46}$ The downregulation of SIRT3 may inhibit cell growth and proliferation, as well as reducing tumor burden in vivo. The apoptotic cell death, anoikis, triggered by loss of extracellular matrix contacts, is dysregulated in many chronic debilitating and fatal diseases, including cancer. SIRT3 may have a role in meditating anoikis 
resistance and carcinogenesis as it is highly expressed via regulating a CD95/Fas-mediated signaling pathway in oral squamous cell carcinoma (OSCC) cells, which exhibit a greater tumor burden. ${ }^{47}$ SIRT3 is overexpressed to a greater extent in several human oral cancer cells and tissues than in normal controls, and downregulation of SIRT3 in these cells can inhibit OSCC cell growth and proliferation as well as enhancing radiotherapeutic and chemotherapeutic drug cytotoxicity. Receptor-interacting protein (RIP) can shuttle between Fas-mediated cell death and focal adhesion kinase (FAK)-mediated survival pathways. It has been demonstrated that SIRT3 might engage in cross-talk with Fas/RIP/FAK death-survival pathways in OSCC development. ${ }^{48}$ Moreover, downregulation of SIRT3 can inhibit acetylation of nicotinamide mononucleotide adenylyltransferase 2 (NMNAT2) and $\mathrm{NAD}^{+}$synthesis activity of the enzyme. The low expression of SIRT3 significantly promotes apoptosis, thereby inhibiting mitotic entry, growth and proliferation of non-small cell lung carcinoma cells, which is related to energy metabolism involved in the interaction between SIRT3 and NMNAT2. ${ }^{49}$ Therefore, SIRT3 induces survival and protects several cell types from cellular damage by maintaining mitochondrial integrity and function or by enhancing their resistance to stress-mediated cell death. In addition, the overexpression of SIRT3 in cancer may result in increase of survival signals and suppression of apoptotic signals, thus enhancing carcinogenesis (Figure 4).

\section{SIRT3 Functions as a Tumor Suppressor}

Conversely, a pro-apoptotic role for SIRT3 in cancers has been revealed. First, SIRT3 is required for apoptosis induced by selectively silencing $\mathrm{Bcl}-2$ in HCT116 human epithelial cancer cells, and it also functions in c-Jun N-terminal kinase 2
(JNK2)-regulated apoptosis. Thus the pro-apoptotic functioning of SIRT3 is selectively coupled with defined pathways regulating cell survival under basal conditions. ${ }^{50}$ In chronic myelogenous leukemia K562 and promyelocitic human leukemia U937 cells, treated by Kaempferol, which is a flavonoid with antioxidant and pro-oxidant activity present in various natural sources, can result in the inactivation of Akt and the activation of the mitochondrial phase of the apoptotic program with an increase of Bax and SIRT3, decrease of Bcl2 , release of cytochrome $c$, activation of caspase- 3 and cell death, suggesting a tumor suppressor role of SIRT3. ${ }^{51}$ Mechanistically, SIRT3 mediates metabolic reprogramming by destabilizing HIF- $1 \alpha$, a transcription factor that controls glycolytic gene expression. The loss of SIRT3 increases ROS production, leading to HIF-1 $\alpha$ stabilization. Thus, SIRT3 expression is reduced in human breast cancers, and its loss correlates with the upregulation of HIF-1 $\alpha$ target genes. ${ }^{52}$ Moreover, SIRT3 can act as a tumor suppressor via suppressing ROS and regulating HIF-1 $\alpha$. Primary mouse embryo fibroblasts or tumor cells expressing SIRT3 shorthairpin RNA may exhibit a greater potential to proliferate, and augment HIF-1 $\alpha$ protein stabilization and transcriptional activity in hypoxic conditions. The knockdown of SIRT3 may also increase carcinogenesis in xenograft models, which is abolished by providing the antioxidant $N$-acetyl cysteine. ${ }^{37}$ Furthermore, SIRT3 expression can be downregulated in human hepatocellular carcinoma (HCC) cells. The overexpression of SIRT3 can also reduce the intracellular NAD ${ }^{+}$ level, repress the extracellular signal-regulated kinase 1/2 signaling pathway, activate the Akt and JNK signaling pathway and upregulate p53 protein level through downregulation of Mdm2 to slow down p53 degradation, suggesting that SIRT3 may have an important role in HCC development as well as progression, and may be a promising therapeutic

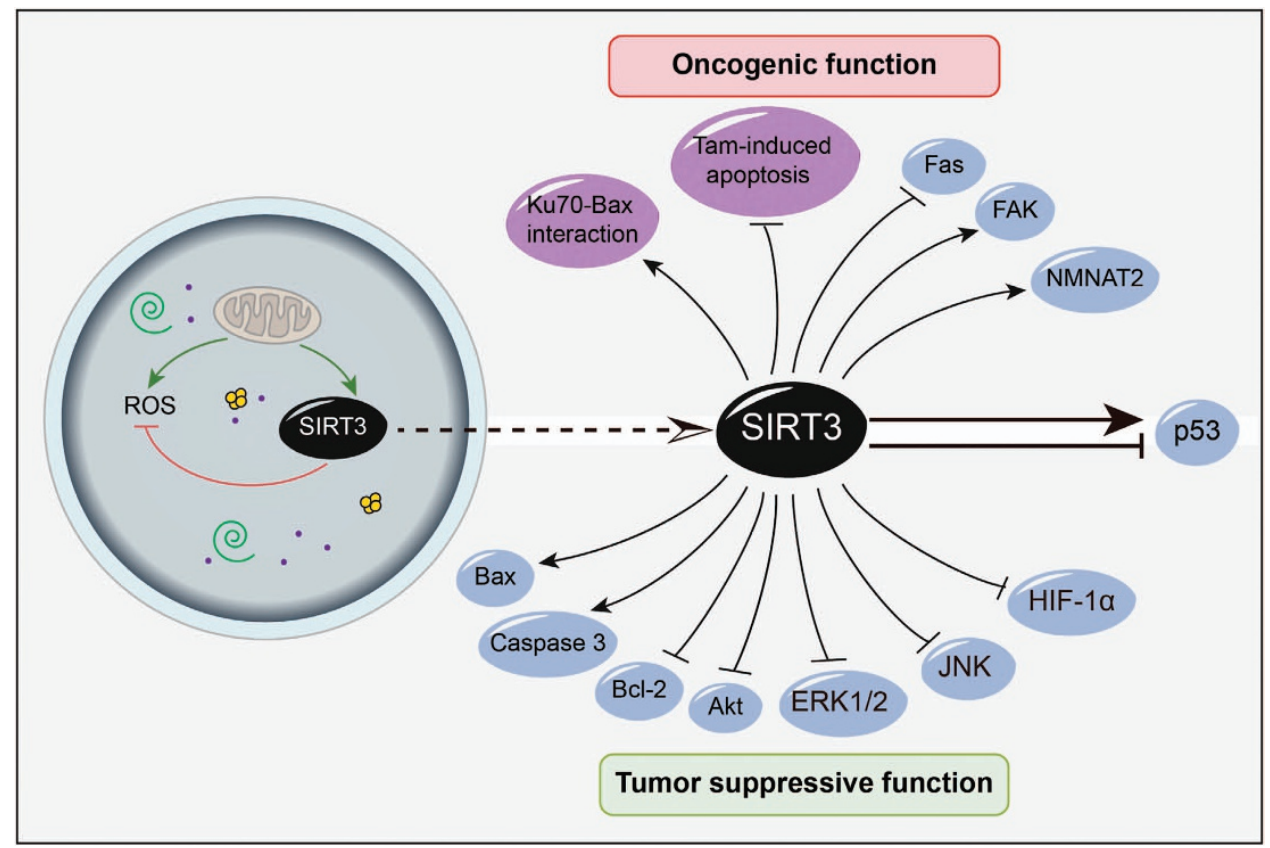

Figure 4 SIRT3 has as an oncogenic or a tumor-suppressive role in cancer 
target for HCC. ${ }^{53}$ The expression of SIRT3 can be downregulated in human lung adenocarcinoma cells when compared with that in adjacent normal cells. In A549 lung adenocarcinoma cells, overexpression of SIRT3 can induce apoptosis, increase $\mathrm{Bax} / \mathrm{Bcl}-2$ and $\mathrm{Bad} / \mathrm{Bcl}-\mathrm{X}_{\mathrm{L}}$ ratios, and promote AIF translocation to the nucleus. Also, SIRT3 upregulation leads to increased p53 and p21 protein levels as well as decreased intracellular ROS levels. Thus, SIRT3 may be a tumor suppressor in lung adenocarcinoma development and progression. ${ }^{54}$ Moreover, SIRT3 can deacetylate cyclophilin D, a protein required for hexokinase II (HK II) binding to voltage-dependent anion channels to keep mitochondrial integrity. In some cancer cells, SIRT3 induces HK II to dissociate from the mitochondria and activate apoptosis. Notably, the expression level of SIRT3 is inversely correlated with clinical-pathological variable, including tumor infiltration, tumor differentiation and 5-year survival of gastric cancer (GC) patients. Knockdown of SIRT3 can significantly increase the expression of HIF-1 $\alpha$, suggesting that SIRT3 may function as a mitochondrial tumor suppressor in GC. ${ }^{55}$ As mentioned above, SIRT3 seems to function as a pro-apoptotic signal in some cancer cells, thus guarding the cell as a tumor suppressor (Figure 4).

\section{SIRT3, a Therapeutic Target in Cancer}

The fact that SIRT3 can regulate cancer processes may implicate SIRT3 as a potential therapeutic target in cancer treatment. ${ }^{56}$ To our knowledge, there are no published reports on clinical trials using class III HDAC inhibitors of SIRT3 to treat cancer. However, class III HDAC activators of SIRT3 such as resveratrol are currently in early stages of clinical trials, which have been tested for safety and potential treatment of cancer. Resveratrol, a polyphenol phytoalexin found in the skin of red grapes, has recently been reported to act as a SIRT3 activator and possess diverse natural therapeutic benefits, including cardiac protection, anti-inflammatory and anticarcinogenic effects, preventing obesity and promoting longevity. ${ }^{57}$ These therapeutic benefits seem to work, at least in part, by activating SIRT3, although it is not yet clear whether these effects are mediated by direct or indirect mechanisms. Moreover, resveratrol is the most studied SIRT3 activator in cancer prevention in neuroblastoma, hepatoma, breast, lung, pancreatic and prostate cancers. ${ }^{58}$ As a major active component of the Chinese traditional medicinal plant Scutellaria baicalensis Georgi, oroxylin A has been reported as a potential anticancer drug. Oroxylin $A$ inhibits the glycolysis and the binding of HK II with mitochondria in human breast carcinoma cells, which is dependent on SIRT3. The level of SIRT3 in mitochondria is increased by oroxylin A. Then, SIRT3 deacetylates cyclophilin D, diminishes its peptidyl-prolyl cis-trans isomerase activity and induces its dissociation from the adenine nucleotide translocation. Finally, SIRT3-induced inactivation of cyclophilin D leads to the detachment of mitochondrial HK II and the inhibition of glycolysis. ${ }^{59}$ Also, under the guidance of a virtual screening workflow based on shape matching and docking, a novel SIRT3 inhibitor with scaffolds, with the name of 5-amino-2-phenyl-benzoxazole has been discovered, which may provide a good starting point for future development of SIRT3 inhibitors with a novel structural scaffold. ${ }^{60}$

\section{Conclusions}

SIRT3 is a mitochondrial deacetylase that may act on numerous substrates to activate fat oxidation, amino-acid metabolism and electron transport. By targeting more than half a dozen key metabolic enzymes, SIRT3 is perfectly positioned to orchestrate coordinated shifts in mitochondrial metabolism, with potential implications for diseases especially cancer. We are just starting to appreciate the dual roles of SIRT3 in treating cancer, and the current controversy regarding its roles in cancer emphasizes the importance of examining this area further. SIRT3 can function either as a tumor promoter or as a tumor suppressor depending on the cell- and tumor-type, and the presence of different stress or cell death stimuli. As far as we know, several studies have implicated SIRT3 as a novel therapeutic target, but the relationship between SIRT3 and cancer is controversial. Therefore, understanding the mechanistic differences in cancer types will enhance our knowledge of the oncogenic and tumor-suppressive roles of SIRT3 and thus, ultimately help in developing a novel cancer therapeutic strategy.

\section{Conflict of Interest}

The authors declare no conflict of interest.

Acknowledgements. We are grateful to Dr. B Liu and Prof. CH Huang (Sichuan University) for their critical reviews on this manuscript. We also thank Prof. R Kasimu and Prof. JH Wang (Xinjiang Medical University) for their constructive discussion on this work. This work was supported by grants from the National 973 Basic Research Program of China (Nos. 2010CB529900 and 2013CB911300), the Key Projects of the National Science and Technology Pillar Program (No. 2012BAl30B02), National Natural Science Foundation of China (Nos. U1170302, 81260628, 81303270 and 81202403), Shenyang Science and Technology Project (No. F12-157-9-00), Shenyang Pharmaceutical University scientific research fund (No. ZCJJ2013407) and Autonomous Region of Xinjiang Project of Science and Technology (No. 201191259).

1. Weir HJ, Lane JD, Balthasar N. SIRT3: a central regulator of mitochondrial adaptation in health and disease. Genes Cancer 2013; 4: 118-124.

2. Hallows WC, Albaugh BN, Denu JM. Where in the cell is SIRT3?-Functional localization of an NAD +-dependent protein deacetylase. Biochem J 2008; 411: e11-e13.

3. Buler M, Aatsinki SM, Izzi V, Hakkola J. Metformin reduces hepatic expression of SIRT3, the mitochondrial deacetylase controlling energy metabolism. PLoS One 2012; 7: e49863.

4. Hallows WC, Lee S, Denu JM. Sirtuins deacetylate and activate mammalian acetyl-CoA synthetases. Proc Natl Acad Sci USA 2006; 103: 10230-10235.

5. Schlicker C, Gertz M, Papatheodorou P, Kachholz B, Becker CF, Steegborn C. Substrates and regulation mechanisms for the human mito-chondrial sirtuins Sirt3 and Sirt5. J Mol Biol 2008; 382: 790-801.

6. Ahn BH, Kim HS, Song S, Lee IH, Liu J, Vassilopoulos A et al. A role for the mitochondrial deacetylase Sirt3 in regulating energy homeostasis. Proc Natl Acad Sci USA 2008; 105: 14447-14452.

7. Yang Y, Cimen H, Han MJ, Shi T, Deng JH, Koc H et al. NAD + -dependent deacetylase SIRT3 regulates mito- chondrial protein synthesis by deacetylation of the ribosomal protein MRPL10. J Biol Chem 2010; 285: 7417-7429.

8. Shulga N, Pastorino JG. Ethanol sensitizes mitochondria to the permeability transition by inhibiting deacetylation of cyclophilin-D mediated by sirtuin-3. J Cell Sci 2010; 123: $4117-4127$.

9. Shi T, Wang F, Stieren E, Tong Q. SIRT3, a mito- chondrial sirtuin deacetylase, regulates mito- chondrial function and thermogenesis in brown adipocytes. J Biol Chem 2005; 280: 13560-13567. 
10. Sundaresan NR, Gupta M, Kim G, Rajamohan SB, Isbatan A, Gupta MP. Sirt3 blocks the cardiac hypertrophic response by augmenting Foxo3a- dependent antioxidant defense mechanisms in mice. J Clin Invest 2009; 119: 2758-2771.

11. Finley LW, Haigis MC. Metabolic regulation by SIRT3: implications for tumorigenesis. Trends Mol Med 2012; 18: 516-523.

12. Alhazzazi TY, Kamarajan P, Verdin E, Kapila YL. SIRT3 and cancer: tumor promoter or suppressor? Biochim Biophys Acta 2011; 1816: 80-88.

13. Bruzzone S, Parenti MD, Grozio A, Ballestrero A, Bauer I, Del Rio A et al. Rejuvenating sirtuins: the rise of a new family of cancer drug targets. Curr Pharm Des 2013; 19 614-623.

14. Nguyen GT, Schaefer S, Gertz M, Weyand M, Steegborn C. Structures of human sirtuin 3 complexes with ADP-ribose and with carba-NAD + and SRT1720: binding details and inhibition mechanism. Acta Crystallogr D Biol Crystallogr 2013; 69: 1423-1432.

15. Schwer B, Bunkenborg J, Verdin RO, Andersen JS, Verdin E. Reversible lysine acetylation controls the activity of the mitochondrial enzyme acetyl-CoA synthetase 2. Proc Natl Acad Sci USA 2006; 103: 10224-10229.

16. Sanders BD, Zhao K, Slama JT, Marmorstein R. Structural basis for nicotinamide inhibition and base exchange in Sir2 enzymes. Mol Cell 2007; 25: 463-472.

17. Wen X, Wu J, Wang F, Liu B, Huang C, Wei Y. Deconvoluting the role of reactive oxygen species and autophagy in human diseases. Free Radic Biol Med 2013; 65C: 402-410.

18. Bause AS, Haigis MC. SIRT3 regulation of mitochondrial oxidative stress. Exp Geronto 2013; 48: 634-639.

19. Bell EL, Guarente L. The SirT3 divining rod points to oxidative stress. Mol Cell 2011; 42 561-568.

20. Hirschey MD, Shimazu T, Huang JY, Schwer B, Verdin E. SIRT3 regulates mitochondria protein acetylation and intermediary metabolism. Cold Spring Harb Symp Quant Biol 2011; 76: $267-277$

21. Park SH, Ozden O, Jiang H, Cha YI, Pennington JD, Aykin-Burns N et al. Sirt3, Mitochondrial ROS, Ageing, and Carcinogenesis. Int J Mol Sci 2011; 12: 6226-6239.

22. Haigis MC, Deng CX, Finley LW, Kim HS, Gius D. SIRT3 is a mitochondrial tumor suppressor: a scientific tale that connects aberrant cellular ROS, the Warburg effect, and carcinogenesis. Cancer Res 2012; 72: 2468-2472.

23. Han C, Someya S. Maintaining good hearing: Calorie restriction, Sirt3, and glutathione. Exp Gerontol 2013; 48: 1091-1095.

24. Opalach K, Rangaraju S, Madorsky I, Leeuwenburgh C, Notterpek L. Lifelong calorie restriction alleviates age-related oxidative damage in peripheral nerves. Rejuvenation Res 2010: 13: 65-74.

25. Hebert AS, Dittenhafer-Reed KE, Yu W, Bailey DJ, Selen ES, Boersma MD et al. Calorie restriction and SIRT3 trigger global reprogramming of the mitochondrial protein acetylome. Mol Cell 2013; 49: 186-199.

26. Onyango P, Celic I, McCaffery JM, Boeke JD, Feinberg AP. SIRT3, a human SIR2 homologue, is an NAD-dependent deacetylase localized to mitochondria. Proc Natl Acad Sci USA 2002; 99: 13653-13658.

27. Schwer B, North BJ, Frye RA, Ott M, Verdin E. The human silent information regulator (Sir)2 homologue hSIRT3 is a mitochondrial nicotinamide adenine dinucleotide-dependent deacetylase. J Cell Biol 2002; 158: 647-657.

28. Kong $X$, Wang $R$, Xue $Y$, Liu $X$, Zhang $H$, Chen $Y$ et al. Sirtuin 3 , a new target of PGC-1alpha, plays an important role in the suppression of ROS and mitochondrial biogenesis. PLoS One 2010; 5: e11707.

29. Giralt A, Hondares E Villena JA, Ribas F, Díaz-Delfín J, Giralt M et al Peroxisome proliferator-activated receptor-gamma coactivator-1alpha controls transcription of the Sirt3 gene, an essential component of the thermogenic brown adipocyte phenotype. J Biol Chem 2011; 286: 16958-16966.

30. Osborne B, Cooney GJ, Turner N. Are sirtuin deacylase enzymes important modulators o mitochondrial energy metabolism? Biochim Biophys Acta 27August 2013; doi:10.1016/ bbagen.2013.08.016.

31. Guarente L. Introduction: sirtuins in aging and diseases. Methods Mol Biol 2013; 1077 3-10.

32. Hanahan D, Weinberg RA. Hallmarks of cancer: the next generation. Cell 2011; 144 646-674.

33. Tao R, Coleman MC, Pennington JD, Ozden O, Park SH, Jiang $\mathrm{H}$ et al. Sirt3-mediated deacetylation of evolutionarily conserved lysine 122 regulates $M n S O D$ activity in response to stress. Mol Cell 2010; 40: 893-904.

34. Scher MB, Vaquero A, Reinberg D. SirT3 is a nuclear NAD +-dependent histone deacetylase that translocates to the mitochondria upon cellular stress. Genes Dev 2007; 21: $920-928$

35. Schumacker PT. SIRT3 controls cancer metabolic reprogramming by regulating ROS and HIF. Cancer Cell 2011; 19: 299-300.

36. Koyama T, Kume S, Koya D, Araki S, Isshiki K, Chin-Kanasaki M et al. SIRT3 attenuate palmitate-induced ROS production and inflammation in proximal tubular cells. Free Radic Biol Med 2011; 51: 1258-1267.
37. McGuinness D, McGuinness DH, McCaul JA, Shiels PG. Sirtuins, bioageing, and cancer. J Aging Res 2011; 2011: 235754.

38. Semenza GL. Hypoxia-inducible factors: media- tors of cancer progression and targets for cancer therapy. Trends Pharmacol Sci 2012; 33: 207-214.

39. Bell EL, Emerling BM, Ricoult SJ, Guarente L. SirT3 suppresses hypoxia inducible factor 1 alpha and tumor growth by inhibiting mitochondrial ROS production. Oncogene 2011; 30: $2986-2996$.

40. Cheng Y, Ren X, Gowda AS, Shan Y, Zhang L, Yuan YS et al. Interaction of Sirt3 with OGG1 contributes to repair of mitochondrial DNA and protects from apoptotic cell death under oxidative stress. Cell Death Dis 2013; 4: e731.

41. Alhazzazi TY, Kamarajan P, Verdin E, Kapila YL. Sirtuin-3 (SIRT3) and the Hallmarks of Cancer. Genes Cancer 2013: 4: 164-171.

42. Lai CC, Lin PM, Lin SF, Hsu CH, Lin HC, Hu ML et al. Altered expression of SIRT gene family in head and neck squamous cell carcinoma. Tumour Biol 2013; 34: 1847-1854.

43. Sundaresan NR, Samant SA, Pillai VB, Rajamo- han SB, Gupta MP. SIRT3 is a stress responsive deacetylase in cardiomyocytes that protects cells from stress-mediated cell death by deacetylation of Ku-70. Mol Cell Biol 2008; 28: 6384-6401.

44. Li S, Banck M, Mujtaba S, Zhou MM, Sugrue MM, Walsh MJ. p53-induced growth arrest is regulated by the mitochondrial SirT3 deacetylase. PLoS One 2010; 5: e10486.

45. Zhang L, Ren X, Cheng Y, Huber-Keener K, Liu X, Zhang Y et al. Identification of Sirtuin 3, a mitochondrial protein deacetylase, as a new contributor to tamoxifen resistance in breast cancer cells. Biochem Pharmacol 2013; 86: 726-733.

46. Zhao Y, Yang H, Wang X, Zhang R, Wang C, Guo Z. Sirtuin-3 (SIRT3) expression is associated with overall survival in esophageal cancer. Ann Diagn Pathol 2013; 17: 483-485

47. Kamarajan P, Alhazzazi TY, Danciu T, D'silva NJ, Verdin E, Kapila YL. Receptorinteracting protein (RIP) and Sirtuin-3 (SIRT3) are on opposite sides of anoikis and tumorigenesis. Cancer 2012; 118: 5800-5810.

48. Alhazzazi TY, Kamarajan P, Joo N, Huang JY, Verdin E, D'Silva NJ et al. Sirtuin-3 (SIRT3), a novel potential therapeutic target for oral cancer. Cancer 2011; 117: 1670-1678

49. Li H, Feng Z, Wu W, Li J, Zhang J, Xia T. SIRT3 regulates cell proliferation and apoptosis related to energy metabolism in non-small cell lung cancer cells through deacetylation of NMNAT2. Int J Oncol 2013; 43: 1420-1430.

50. Allison SJ, Milner J. SIRT3 is pro-apoptotic and participates in distinct basal apoptotic pathways. Cell Cycle 2007; 6: 2669-2677.

51. Marfe G, Tafani M, Indelicato M, Sinibaldi-Salimei P, Reali V, Pucci B et al. Kaempferol induces apoptosis in two different cell lines via Akt inactivation, Bax and SIRT3 activation, and mitochondrial dysfunction. J Cell Biochem 2009; 106: 643-650.

52. Finley LW, Carracedo A, Lee J, Souza A, Egia A, Zhang J et al. SIRT3 opposes reprogramming of cancer cell metabolism through $\mathrm{HIF1} \alpha$ destabilization. Cancer Cell 2011; 19: 416-428.

53. Zhang YY, Zhou LM. Sirt3 inhibits hepatocellular carcinoma cell growth through reducing Mdm2-mediated p53 degradation. Biochem Biophys Res Commun 2012; 423: 26-31.

54. Xiao K, Jiang J, Wang W, Cao S, Zhu L, Zeng H et al. Sirt3 is a tumor suppressor in lung adenocarcinoma cells. Oncol Rep 2013; 30: 1323-1328.

55. Yang B, Fu X, Shao L, Ding Y, Zeng D. Aberrant expression of SIRT3 is conversely correlated with the progression and prognosis of human gastric cancer. Biochem Biophys Res Commun 2014; 443: 156-160.

56. Liu B, Wen X, Cheng Y. Survival or death: disequilibrating the oncogenic and tumor suppressive autophagy in cancer. Cell Death Dis 2013; 4: e892.

57. Signorelli $P$, Ghidoni R. Resveratrol as an anticancer nutrient: molecular basis, open questions and promises. J Nutr Biochem 2005; 16: 449-466.

58. Rayalam S, Yang JY, Ambati S, Della-Fera MA, Baile CA. Resveratrol induces apoptosis and inhibits adipogenesis in 3T3-L1 adipocytes. Phytother Res 2008; 22: $1367-1371$

59. Wei L, Zhou Y, Dai Q, Qiao C, Zhao L, Hui H et al. Oroxylin A induces dissociation of hexokinase II from the mitochondria and inhibits glycolysis by SIRT3-mediated deacetylation of cyclophilin D in breast carcinoma. Cell Death Dis 2013; 4: e601.

60. Salo HS, Laitinen T, Poso A, Jarho $E$, Lahtela-Kakkonen $M$. Identification of nove SIRT3 inhibitor scaffolds by virtual screening. Bioorg Med Chem Lett 2013; 23: 2990-2995.

Cell Death and Disease is an open-access journal
published by Nature Publishing Group. This work is
licensed under a Creative Commons Attribution-NonCommercial-
ShareAlike 3.0 Unported License. To view a copy of this license, visit
http://creativecommons.org/licenses/by-nc-sa/3.0/

